\title{
Prognostic Value of Serum Uric Acid Levels to the Cardiovascular Events in Hypertensives
}

\author{
MV Papavasileiou ${ }^{1}$, G Moustakas ${ }^{1}$, A Karamanou ${ }^{1 *}$, SPatsianis ${ }^{1}$, D Aravantinos ${ }^{1}$ and A Pittaras ${ }^{2}$ \\ ${ }^{1}$ Sismanoglion GH Cardiology, Antihypertensive clinic Athens, Greece \\ ${ }_{2}^{2}$ Mediton Cardiology Diagnostic Center, Athens, Greece
}

*Corresponding author: A Karamanou, Sismanoglion GH Cardiology,

Received Date: January 04, 2021

Antihypertensive clinic Athens, Greece.

Published Date: February 15, 2021

\begin{abstract}
Aim: The aim of the study was to investigate the association of serum uric acid (SUA) levels with the major cardiovascular events (MACE) in hypertensive patients.

Methods and Materials: This is a prospective analysis including 652 hypertensive patients ( $52,6 \%$ female) of mean age at the entry: $57,2 \pm 13,1$ years, mean office systolic/diastolic blood pressure (S/DBPo): 152,3 $\pm 19,1 / 94,4 \pm 11,83 \mathrm{mmHg}$, mean office heart rate (HRo): 76,0 $\pm 12 \mathrm{beats} / \mathrm{min}$, treated or newly diagnosed untreated. The median follow-up period was 9 years. SUA were measured at the entry of the study. Major cardiovascular events (MACE) (myocardial infarction, unstable angina, transient ischemic attack or stroke, peripheral vascular intervention, heart failure events, cardiovascular death) were registered. We estimated the prognostic value of SUA to the MACE. Cox proportional hazard model was employed to determine the prognostic value of uric acid.
\end{abstract}

Result: The median follow-up period was 9 years. There were 264 (40,5\%) MACE at the end of the study. Cox regression statistical analysis revealed that SUA was a strong predictor of MACE (HR: 1,14, 95\%CI: 1,06 - 1,2 p-value=0,000)

Conclusion: SUA levels have statistically significant prognostic value to MACE in hypertensive patients.

\section{Introduction}

Uric acid is a product of the activity of xanthine oxidase, an enzyme increasingly implicated as a mechanistic participant in oxidant stress and cardiovascular disease. Contributing factors responsible for hyperuricemia are alcoholic and high-purine foods consumption,lowwater consumptionand poorly exercising. In adults with essential hypertension an association with hyperuricemia is very common. Prevalence of hyperuricemia is $30 \%$ in untreated hypertensives and $>75 \%$ in malignant hypertension. Uric acid is thought to play a pathogenic role in hypertension [1-3] mediated by several mechanisms such as inflammation, vascular smooth muscle cell proliferation in renal microcirculation, endothelial dysfunction, and activation of the renin - angiotensin - aldosterone system [4-7]. Animal models have shown that acute elevations of serum urate, by inhibition of uricase, induce a prompt rise in blood pressure and that chronic urate elevation maintains the rise in pressure and induces irreversible vascular damage and glomerular changes, and results in a form of salt-sensitive hypertension [8, 9]. Furthermore, studies have shown that in overweight and obese subjects, hyperinsulinemia secondary to insulin resistance may enhance their absorption of uric acid and thus contribute to the association of hyperuricemia with hypertension [10].

Also, numerous studies have noted an association of elevated serum uric acid (SUA) levels also with heart failure [11], coronary artery disease [12] and stroke [13]. Several pathophysiological mechanisms have been postulated including multiple proatherogenic processes, increased oxidative stress [14, 15], 
vascular smooth muscle cell proliferation [16], leukocyte activation [17], platelet adhesiveness and aggregation [18] and crystal formation within coronary atherosclerotic plaques [19]. Xanthine oxidase activity is increased during ischemia and heart failure, and treatment with xanthine oxidase inhibitors has favorable effects on myocardial oxygen consumption and endothelium- dependent vascular function $[20,21]$. Purpose of this study was to evaluate the predictive role of SUA levels with respect MACE (myocardial infarction, stroke, cardiovascular death) in a large Greek cohort of hypertensive men and women, in whom the SUA levels were routinely obtained at baseline.

\section{Patient and Method}

\section{Patient Population}

We studied 652 consecutive hypertensives (52,6\% female) of mean age $57,2 \pm 13,1$ years, treated or newly diagnosed nevertreated with anti-hypertensive drugs who were self-referred to our outpatient cardiology hypertensive clinic for BP evaluation. The median follow-up period was 9(5-14) years. Patients were excluded from the study, if they suffered from any cardiovascular disease, secondary hypertension, and any other clinically significant concurrent medical condition such as thyroidal, psychiatric, neuromuscular, chronic kidney disease, respiratory, hepatic or gastrointestinal illness, or systemic disease. None of the participants had any history or clinical/laboratory evidence of recent infection, inflammation or underwent any medical treatment (including antiinflammatory treatment and hormone replacement therapy) the last month before entry into the study. Patients under treatment for hyperuricemia were also excluded from the study. The primary endpoint of the trial was combined events of myocardial infarction or unstable angina, transient ischemic attack or stroke, peripheral vascular intervention, heart failure events or cardiovascular death during the study period. The study was approved by our hospital's ethics committee and conformed to the 1964 Declaration of Helsinki. All subjects gave their written informed consent at the baseline of our study.

\section{Baseline Measurements}

At baseline, all participants were individually interviewed and information on gender, age, weight, height, waist circumference, hip circumference, smoking status, physical exercise status and diet was recorded. Body mass index (BMI) and waist to hip ratio (WHR) determined. Venous blood samples were drawn from all participants after an overnight fast (8-12 h) for the determination of SUA, fasting glucose (glo), lipids and serum creatinine ( $\mathrm{sCr}$ ). At baseline, resting sitting office BP was measured twice, using Cuff's of a size appropriated to the arm circumference, with at least $5 \mathrm{~min}$ intervals using an automatic sphygmomanometer. If the difference between the first and second measurement was $>10 \mathrm{~mm} \mathrm{Hg}$, then repeated measurements were performed. The average of the last two measurements was used for screening. Moreover, a bilateral measurement was performed to define the arm subjected to the relatively higher hemodynamic load and accordingly was used for all the following measurements.

\section{Outcomes Measure}

Follow-up data, based on interviews, health care facility medical records, and death certificates, were collected during the follow up period. Cardiovascular death was ascertained by either certificate, a proxy interview, or both. In the former case, the underlying cause of death was coded according to the International Classification of Disease, Ninth-tenth version (ICD-9 and ICD-10). Years of follow-up for each patient were calculated from baseline to the date of death for decedents and to the date of follow-up for those still alive.

\section{Statistical Analysis}

Continuous variables are presented as either mean $( \pm$ standard deviation) or medians (interquartile range), and categorical variables as percentages. The significance of differences in baseline characteristics of the participants according to SUA were compared using Pearson's Chi-square test or Student's t-tests, as appropriate. Correlation analyses were performed using Pearson's correlation coefficient. Cox proportional hazard regression models were used to estimate the hazard ratio (HR) and 95\% confidence interval (CI) for the effect of baseline SUA. To assess independent associations of SUA with outcomes, covariates were considered confounders and were entered into the summary model. Statistical significance was set at $p$ value $<0,05$. Results were analyzed with SPSS for Windows software.

\section{Result}

Clinical and laboratory characteristics of the study population are shown in (Table 1). The mean age of the study population at baseline was 57,2 year old (52.6\% females) and the mean BMI was $28,0 \pm 4,3 \mathrm{~kg} / \mathrm{m} 2$, median W/H: 0,88 (0,84-0,95). Moreover 57,8 \% patients were newly diagnosed hypertensives. The mean serum glo was $104,3 \pm 31,6 \mathrm{mg} / \mathrm{dl}$ and the mean $\mathrm{sCr}$ was $0,95 \pm 0,4 \mathrm{mg} / \mathrm{dl}$. Moreover, mean SUA level was $5,1 \pm 1,7 \mathrm{mg} / \mathrm{dl}$ (Table 1). During a median 9(5-14) years of follow-up, $270(41,4 \%)$ persons of the study population developed MACE events. A total of 85 patients, (13\%) of the study population, developed myocardial infarction (MI), or unstable angina, 102 patients (15,6\%) developed transient ischemic attack (TIA) or stroke, 13 patients $(1,99 \%)$ had hospitalization for heart failure, 12 patients $(1,84 \%)$ underwent peripheral vascular intervention and 58 patients $(8,89 \%)$ died from cardiovascular causes (Table 2) The median age at the time of occurring MACE was 67 (59-75) years old. Males had statisticaly significant increase risk of developing MACE compared to females Moreover, treated patients at baseline did not differ as far as it concern occurring MACE, compared to newly diagnosed nevertreated with anti-hypertensive drugs patients at baseline.

According to chi-square test hypertensive patients on treatment had statistically significant more cardiovascular events compared to hypertensive patients without treatment $(\mathrm{p}<0,001)$. Additionally, 
male hypertensive patients had significantly more cardiovascular events compared to female $(p<0,001)$ (Table 3$)$. At baseline, higher serum uric acid levels were associated with age $(r=0,085, p=0,029)$, BMI $(r=0,100, p<0,011)$, waist $(r=0,247, p<0,001)$, waist to hip

Table 1: Demographic and clinical characteristics of the study population at baseline $(n=652)$.

\begin{tabular}{|c|c|}
\hline Baseline age (years) & $57,2 \pm 13,1$ \\
\hline Male (\%) & 47,4 \\
\hline Treated (\%) & 42,2 \\
\hline BMI $\left(\mathrm{kg} / \mathrm{m}^{2}\right)$ & $28,0 \pm 4,3$ \\
\hline Waist (cm) & $98(89-105)$ \\
\hline Hip (cm) & $108(102-115)$ \\
\hline Waist to Hip ratio (W/H) & $0,88(0,84-0,95)$ \\
\hline Serum urea (mg/dl) & $41,7 \pm 15.9$ \\
\hline Serum creatinine $(\mathrm{mg} / \mathrm{dl})$ & $0,95 \pm 0,34$ \\
\hline e-GFR (mL/min/1,73²) & $81,7 \pm 35,7$ \\
\hline Serum uric acid (mg/dl) & $5,18 \pm 1,7$ \\
\hline Serum glucose (mg/dl) & $104,3 \pm 31,6$ \\
\hline Total cholesterol (mg/dl) & $232,9 \pm 49,7$ \\
\hline HDL cholesterol (mg/dl) & $51,4 \pm 14,1$ \\
\hline LDL cholesterol (mg/dl) & $156,4 \pm 47,1$ \\
\hline Triglycerids (mg/dl) & $123,5 \pm 71,4$ \\
\hline SBPo (mmHg) & $152,3 \pm 19,1$ \\
\hline DBPo (mmHg) & $94,4 \pm 11,83$ \\
\hline HRo (bpm) & $76,0 \pm 12$ \\
\hline Event age (years) & $67(59-75)$ \\
\hline $\begin{array}{c}\text { BMI: Body Mass Index, SBPo: office Systolic Blood Pressure, DBPo: office Diastolic Blood } \\
\text { Pressure, HRo: office Heart rate, bpm: beats per minute, e-GFR: estimated clearance creatinine } \\
\text { according to Cockcroft-Gault }\end{array}$ & \\
\hline
\end{tabular}

Table 2: Incidence of MACE during follow-up.

\begin{tabular}{|c|c|c|}
\hline Disease & Number of cases & \% of MACE \\
\hline Total & 270 & 31.4 \\
\hline MI or unstable angina & 85 & 13 \\
\hline TIA or STOKE & 102 & 15,6 \\
\hline Hospitalization for HF & 13 & 4,5 \\
\hline Peripheral Vascular Intervention & 12 & 4,4 \\
\hline Cardiovascular Death & 58 & 1,84 \\
\hline
\end{tabular}

Table 3: Comparison between MACE and No MACE outcome.

\begin{tabular}{|c|c|c|}
\hline & No MACE & MACE value \\
\hline Treated vs no treated, $\%$ & 17,94 vs 38,03 & 24,2 vs 19,78 \\
\hline Male vs female, $\%$ & 22,6 vs 33,28 & 24,69 vs 13,3 \\
\hline
\end{tabular}

Table 4: Correlations of serum uric acid in overall study population $(n=751)$.

\begin{tabular}{|c|c|c|}
\hline & r & p value \\
\hline Age & 0,085 & 0,029 \\
\hline BMI & 0,100 & 0,011 \\
\hline Waist & 0,247 & $<0,001$ \\
\hline Hip & 0,068 & 0,201 \\
\hline Waist to Hip ratio & 0,288 & $<0,001$ \\
\hline
\end{tabular}




\begin{tabular}{|c|c|c|}
\hline SBPo & $-0,034$ & 0,379 \\
\hline DBPo & 0,059 & 0,129 \\
\hline Heart rate o & 0,008 & 0,835 \\
\hline Serum urea & 0,160 & $<0,001$ \\
\hline Serum creatinine & 0,259 & $<0,001$ \\
\hline Creatinine clearance & 0,354 & $<0,001$ \\
\hline Serum glucose & 0,064 & 0,101 \\
\hline $\begin{array}{r}\text { BMI: Body Mass Index, SBPo: office Systolic Blood P } \\
\text { Blood Pressure. }\end{array}$ & & \\
\hline
\end{tabular}

Table 5: Cox regression analysis.

\begin{tabular}{|c|c|c|c|}
\hline & HR & $\mathbf{9 5 \%}$ CI & p value \\
\hline Serum uric acid & 1,13 & $1,04-1,2$ & 0,001 \\
\hline Age & 1,07 & $1,05-1,08$ & $<0,001$ \\
\hline Male gender & 1,48 & $1,07-2,04$ & 0,017 \\
\hline SBPo & 0,996 & $0,99-1,003$ & 0,256 \\
\hline BMI & 1,06 & $0,97-1,039$ & 0,729 \\
\hline Serum glucose & 1,007 & $1,004-1,010$ & $<0,001$ \\
\hline GFR & 1,003 & $0,998-1,008$ & 0,189 \\
\hline
\end{tabular}

Table 6: Hazard ratio for uric acid in entire cohort.

\begin{tabular}{|l|c|c|c|}
\hline & Hazard ratio & $95 \%$ Confidence Intervals & P value \\
\hline Uric Acid $(\mathrm{mg} / \mathrm{dl})$ & 1,13 & $1,04-1,2$ & 0,001 \\
\hline
\end{tabular}

Cox regression analysis showed that serum uric acid, age, male gender and serum fasting glucose were independent predictors for MACE (Table 5). Finally, we found that, in entire cohort, serum 1,004-1,2, p=0,038) (Table 6) (Figure 1).

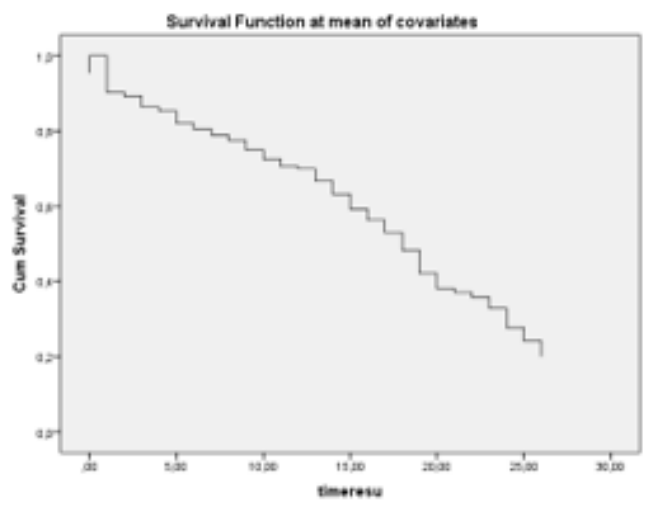

Figure 1: Timeresu: Time from baseline of MACE according to the baseline level of serum uric acid.

\section{Discussion}

The main finding of our study is that elevated serum uric acid is a strong predictor of combined endpoint of myocardial infarction, stroke hospitalization for HF vascular intervention and cardiovascular death in essential hypertensive patients in a long term follow-up. Our findings are in accordance with several other studies and meta-analysis, which have shown that elevated uric acid levels predict an increased risk of cardiovascular events and cardiovascular and all cause mortality [22-27]. Moreover, a systematic review and meta-analysis found that hyperuricaemia may modestly increase the risk of CHD events independently of traditional CHD risk factors. Women were found to have a more pronounced increase in risk for CHD mortality than for men.28 A similar meta-analysis was performed for hyperuricaemia and stroke showing that hyperuricaemia modestly increased the risk of stroke incidence and mortality, independent of known risk factors, but without gender difference [29-31]. 
In contrary, some studies have failed to show uric acid as an independent predictor of death but they were underpowered, included too few events or did not adjust for known confounders [32-34]. Moreover, recent studies of losartan and atorvastatin showed that uric acid reduction contributes to attenuation of cardiovascular risk [35-36]. In a small randomized clinical trial, [37] allopurinol treatment in newly-diagnosed, hypertensive adolescents was associated with significant reductions in casual and 24-hour ambulatory blood pressure compared to placebo. Interestingly, a cohort study of hypouricemic patients enrolled in Veterans Affairs medical centers in the Pacific Northwest reported that the use of allopurinol was associated with a $23 \%$ lower allcause mortality rate [38].

The major strength of our study was that we used a large sample size and excluded for the presence of secondary hypertension, cardiovascular disease, chronic kidney disease, malignancy, and any other medical treatment. However, when interpreting our results, some limitations should be considered. First, bias from follow-up loss may have affected our results. Loss to follow-up is expected, especially in those who are in poor health. However, loss to followup of high-risk people would probably lead to a conservative bias and subsequent underestimation of risk. Furthermore, we were not able to include some important confounders in this study, such as the presence of or use of medication to treat gout and any dietary habits.

\section{Conclusion}

our findings, which were obtained from large cohort of Greek hypertensives, indicated that serum UA may be a predictor for the development of MACE in a large follow-up period, and this association was significant after adjustment for baseline covariates.

\section{Acknowledgement}

None.

\section{Conflict of Interest}

No conflict of interest.

\section{References}

1. Johnson RJ, Feig DI, Herrera-Acosta J, Kang DH (2005) Resurrection of uric acid as a causal risk factor in essential hypertension. Hypertension 45: 18-20.

2. Feig DI, Kang Duk-Hee, Johnson RJ (2008) Uric acid and cardiovascular risk. N Engl J Med 359: 1811-1821.

3. Rudolf P Obermayr, Christian Temml, Georg Gutjahr, Maarten Knechtelsdorfer, Rainer Oberbauer, et al. (2008) Elevated uric acid increases the risk for kidney disease. J Am SocNephrol 19: 2407-2413.

4. Johnson RJ, Kang DH, Feig D, Kivlighn S, Kanellis J, et al. (2003) Is there a pathogenetic role for uric acid in hypertension and cardiovascular and renal disease. Hypertension 41: 1183-1190.

5. Zhang W, Sun K, Yang Y, Zhang H, Hu FB, et al. (2009) Plasma uric acid and hypertension in a Chinese community: prospective study and metaanalysis. ClinChem 55: 2026-2034.

6. Forman JP, Choi H, Curhan GC (2007) Plasma uric acid level and risk for incident hypertension among men. J Am SocNephrol 18: 287-292.
7. Johnson RJ, Feig DI, Herrera-Acosta J, Kang DH (2005) Resurrection of uric acid as a causal risk factor in essential hypertension. Hypertension 45: 18-20.

8. Mazzali M, Kanellis J, Han L, Feng L, Xia YY, et al. (2002) Hyperuricemia induces a primary renal arteriolopathy in rats by a blood pressureindependent mechanism. Am J Physiol Renal Physiol 282(6): F991-F997.

9. Mazzali M, Hughes J, Kim YG, Jefferson JA, Kang DH, et al. (2001) Elevated uric acid increases blood pressure in the rat by a novel crystalindependent mechanism. Hypertension 38(5):1101-1106.

10. Facchini F, Chen YD, Hollenbeck CB, Reaven GM (1991) Relationship between resistance to insulin-mediated glucose uptake, urinary uric acid clearance, and plasma uric acid concentration. JAMA 266: 30083011.

11. He Huang, Baotao Huang, Yulin Li, Yan Huang, Jing Li, et al. (2014) Uric acid and risk of heart failure: a systematic review and meta-analysis. European Journal of Heart Failure 16(1): 15-24.

12. Seo Young Kim, James P Guevara, Kyoung Mi Kim, Hyon K Choi, Daniel F Heitjan, et al. (2010) Hyperuricemia and coronary heart disease: a systematic review and meta-analysis. Arthritis Care and Research 62(2): 170-180.

13. Seo Young Kim, James P Guevara, Kyoung Mi Kim, Hyon K Choi, Daniel F Heitjan, et al. (2009) Hyperuricemia and risk of stroke: a systematic review and meta-analysis. Arthritis and Rheumatism 61(7): 885-892.

14. Bagnati M, Perugini C, Cau C, Bordone R, Albano E, et al. (1999) When and why a water-soluble antioxidant becomes pro-oxidant during copper-induced low-density lipoprotein oxidation: a study using uric acid. Biochem J 340: 143-152.

15. Schlotte V, Sevanian A, Hochstein P, Weithmann KU (1998) Effect of uric acid and chemical analogues on oxidation of human low density lipoprotein in vitro. Free RadicBiol Med 25: 839-847.

16. Rao GN, Corson MA, Berk BC (1998) Uric acid stimulates vascular smooth muscle cell proliferation by increasing platelet-derived growth factor A-chain expression. J BiolChem 266: 8604-8608.

17. Kanellis J, Watanabe S, Li JH, Kang DH, Li P, et al. (2003) Uric acid stimulates monocyte chemo attractant protein-1 production in vascular smooth muscle cells via mitogen-activated protein kinase and cyclooxygenase-2. Hypertension 41: 1287-1293.

18. Johnson R, Kang D, Feig D, Kivlighn S, Kanellis J, et al. (2003) Is there a pathogenetic role for uric acid in hypertension and cardiovascular and renal disease. Hypertension 41(6): 1183-1189.

19. Patetsios P, Rodino W, Wisselink W, Bryan D, Kirwin JD, et al. (1996) Identification of uric acid in aortic aneurysms and atherosclerotic artery. Ann N Y AcadSci 800: 243-245.

20. Marco Canepa, Francesca Viazzi, James B Strait, Pietro Ameri, Roberto Pontremoli, et al. (2017) Longitudinal Association between Serum Uric Acid and Arterial Stiffness: Results from the Baltimore Longitudinal Study of Aging Hypertension 69(2): 228-235.

21. Pacher P, Nivorozhkin A, Szabo C (2006) Therapeutic effects of xanthine oxidase inhibitors: renaissance half a century after the discovery of allopurinol. Pharmacol Rev 58: 87-114

22. Verdecchia P, Schillaci G, Reboldi G, Santeusanio F, Porcellati C, et al. (2000) Relation between serum uric acid and risk of cardiovascular disease in essential hypertension: the PIUMA study. Hypertension 36: 1072-1078.

23. Wang JG, Staessen JA, Fagard RH, Birkenhager WH, Gong L, et al. (2001) Prognostic significance of serum creatinine and uric acid in older Chinese patients with isolated systolic hypertension. Hypertension 37: 1069-1074.

24. Fang J, Alderman MH (2000) Serum uric acid and cardiovascular mortality: the NHANES I epidemiologic follow-up study, 1971- 1992. National Health and Nutrition Examination Survey. JAMA 283: 24042410 
25. Liese AD, Hense HW, Lowel H, Doring A, Tietze M, et al. (1999) Association of serum uric acid with all-cause and cardiovascular disease mortality and incident myocardial infarction in the MONICA Augsburg cohort: World Health Organization Monitoring Trends and Determinants in Cardiovascular Diseases. Epidemiology 10: 391-397.

26. Niskanen LK, Laaksonen DE, Nyyssonen K, Alfthan G, Lakka HM, et al. (2004) Uric acid level as a risk factor for cardiovascular and all-cause mortality in middle-aged men: a prospective cohort study. Arch Intern Med 164: 1546-1551.

27. Tsuneo Konta, Kazunobu Ichikawa, Ryo Kawasaki, Shouichi Fujimoto, Kunitoshi Iseki, Toshiki Moriyama, Kunihiro Yamagata, Kazuhiko Tsuruya, Ichiei Narita, Masahide Kondo, Yugo Shibagaki, Masato Kasahara, Koichi Asahi, Tsuyoshi Watanabe (2020) Association between serum uric acid levels and mortality: a nationwide community-based cohort study Sci Rep 10: 6066.

28. Kim SY, Guevara JP, Kim KM, Choi HK, Heitjan DF, et al. (2010) Hyperuricemia and coronary heart disease: A systematic review and meta-analysis. Arthritis Rheum 62(2): 170-180.

29. Kim SY, Guevara JP, Kim KM, Choi HK, Heitjan DF, et al. (2009) Hyperuricemia and risk of stroke: A systematic review and metaanalysis. Arthritis Rheum 61(7): 885-892.

30. Wang JG, Staessen JA, Fagard RH, Birkenhager WH, Gong L, et al. (2001) Prognostic significance of serum creatinine and uric acid in older Chinese patients with isolated systolic hypertension. Hypertension 37 : 1069-1074.

31. Fang J, Alderman MH (2000) Serum uric acid and cardiovascular mortality: the NHANES I epidemiologic follow-up study, 1971- 1992. National Health and Nutrition Examination Survey. JAMA 283: 24042410.
32. Hu P, Seeman TE, Harris TB, Reuben DB (2001) Is serum uric acid level associated with all-cause mortality in high-functioning older persons: MacArthur studies of successful aging. J Am Geriatr Soc 49: 1587-1742.

33. Culleton BF, Larson MG, Kannel WB, Levy D (1999) Serum uric acid and risk for cardiovascular disease and death: the Framingham Heart Study. Ann Intern Med 131: 7-13.

34. Johannes Nossent, Warren Raymond, Mark Divitini, Matthew Knuiman (2016) Asymptomatic hyperuricemia is not an independent risk factor for cardiovascular events or overall mortality in the general population of the Busselton Health Study. BMC Cardiovasc Disord 16: 256.

35. Athyros V, Mikhailidis D, Liberopoulos E, Kakafika A, Karagiannis A, et al. (2007) Effect of statin treatment on renal function and serum uric acid levels and their relation to vascular events in patients with coronary heart disease and metabolic syndrome. Nephrology Dialysis Transplantation. 22(1): 118-127.

36. Høieggen A, Michael H Alderman, Kjeldsen S, Julius S, Devereux R, et al. (2004) The impact of serum uric acid on cardiovascular outcomes in the LIFE study. Kidney Int 65(3): 1041-1049.

37. Feig D, Soletsky B, Johnson R (2008) Effect of Allopurinol on Blood Pressure of Adolescents With Newly Diagnosed Essential Hypertension. JAMA 300(8): 924-932.

38. Luk A, Levin G, Moore E, Zhou X-H, Kestenbaum B, et al. (2009) Allopurinol and mortality in hyperuricemic patients. Rheumatology 48(7): 804-806. 